\title{
Research on the Power Line Three-stage Over-current Protection Simulation
}

\author{
Yujie Yang ${ }^{\mathrm{a}}$, Liancheng Zhu ${ }^{\mathrm{b}}$, Fuyun $\mathrm{Li}^{\mathrm{c}}$, Zhitao $\mathrm{Wu}^{\mathrm{d}}$ and Changyou Cai ${ }^{\mathrm{e}}$ \\ School of Electronic and Information Engineering, University of Science and Technology Liaoning, \\ China \\ ayangyujie309@163.com, bzhuliancheng@163.com, ${ }^{\mathrm{c}} 306310875 @ q q . c o m,{ }^{d}$ aswzt@163.com, \\ e452839477@qq.com
}

Keywords: MATLAB Simulation, Full Wave Fourier Algorithm, Relay Protection, Three-Stage Over-Current Protection

Abstract: Power line over-current relay protection is an important part of power system, it is an important technical measure to assure the safety and reliable operation of electric power system and electric power line. In this paper, on the basis of the features of the relay protection in the power line, thorough research and the analysis of relay protection both at home and abroad, with the aid of MATLAB/Simulink to build simulation model, Using PSB module to construct a three-stage over-current protection's simulation model of the electric power line, through the analysis of a three-stage over-current relay protection theory, it set up a three-stage over-current protection full-wave Fourier algorithm, and make simulation experiment of protection action. The simulation results show that the simulation analysis can achieve better power line three-stage over-current protection under different kinds of fault simulation and calculation, which can also provide good reference to design the relay protection of power system.

\section{Introduction}

With the rapid development of China's national economy, the power industry keeps evolving towards large-capacity joint grid, thus the performance and reliability requirements for protection devices are increasingly high. In order to improve the safety and reliability of the protection devices, it is important to simulate and study the operating characteristics of power line fault protection in advance and accurately check and analyses it after accidents. Currently, virtual simulation technology of relay protection has become an important method for the development and research of protection devices.

Based on the in-depth study of the basic principles and theories of three-stage over-current protection, this paper expounded the concept of remote backup and close backup in power line relay protection, and proposed the problem of time coordination during three-stage protection action, as well as the coordination problem of three-stage protection when fault occurs in different fault-protected stages. MATLAB/Simulink was used to build simulation models, three-stage over-current protection of power line simulation system was designed, and simulation model was calculated using three-stage over-current protection Fourier algorithm. Through simulation data measurement and simulation waveform analysis, we verified the correctness of theoretical analysis, and provided a good reference for the study of faults in different stages and operating action in three-stage over-current protection.

\section{Three-Stage Over-Current Protection}

Practice shows that the most common types of power line fault are different types of short-circuit fault, which requires the relay protection devices to monitor the circuit and control the breakers to remove the fault. Three-stage over-current protection is the most typical over-current protection of power lines. It includes transient rapid-break over-current protection (stage I protection), time-bound rapid-break over-current protection (stage II protection), and definite time over-current protection 
(stage III protection), and these three protection stages work coordinately. [1]-[4]. The protection range and time characteristics of three-stage over-current protection are shown in Fig. 1.

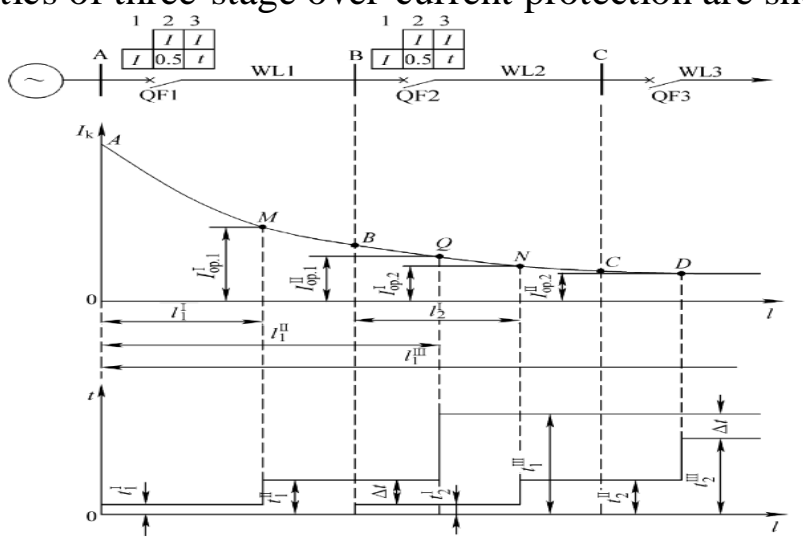

Fig. 1. Scope of protection and time coordination

\section{The Principles of Over-Current Protection}

\section{A. The determination of protection current}

For one-sided power supply lines, over-current protection is installed on the supply side of each lines. When there is a short-circuit on the transmission line, action current is determined by the largest possible circuit to surpass short-circuit at the end of the over-current protected line. In this way, the protection range is limited within the protected lines, thus ensuring the selectivity of the protection, and the fault can be removed immediately. The short-circuit current flowing through the protection installation location [5] as formula (1).

$$
I_{k}=K_{\varphi} E_{\varphi} / Z=K_{\varphi} E_{\varphi} / Z_{s}+Z_{k}
$$

$K_{\varphi}$ is the coefficient related to fault type, when the fault is three-phase fault, it is 1 ; when the fault is two-phase fault, it is $0.866 ; E_{\varphi}$ is the equivalent phasic potential of the power system; $Z$ is the total resistance of the short-circuit power line, and $Z_{s}$ is the total resistance of the power system; $Z_{k}$ is the resistance from the fault point to protection device.

\section{B. The determination of the operation current of rapid-break over-current protection}

When there is a fault in the power line, the current in the line will increase suddenly, and when the current is larger than the designed number of protection current, over-current protection will remove the fault. The protection device which corresponds and acts to the current increase is called over-current protection. Rapid-break over-current protection can be divided into timeless rapid-break over-current protection and time-bound rapid-break over-current protection. Timeless rapid-break over-current protection aims to ensure that in any case only the fault in this line will be removed, and its disadvantage is that it cannot protect the whole line. Thus each line should be equipped a time-bound rapid-break over-current protection to remove other faults in zones that are not protected by timeless rapid-break over-current protection.

The action current of timeless rapid-break over-current protection is determined by the largest possible circuit to avoid short-circuit at the end of the over-current protected zone (point B) as formula (2) [1].

$$
I_{o p 1}^{\prime}=K_{r e l} I_{k . B \cdot \max }^{(3)}
$$

$I_{o p 1}^{\mathrm{I}}$ stands for the action current of timeless rapid-break over-current protection (stage I protection); $K_{r e l}$ stands for the reliability coefficient of timeless rapid-break over-current protection, usually the number is $1.2 \sim 1.3 ; I^{(3)}{ }_{k . B . \max }$ is the short-circuit current at the end of the over-current protected line.

Usually the main protection of electrical equipment is the protection that removes faults with selectivity within the least time. To ensure that the faults can be reliably removed, power lines should 
be equipped with main protection as well as backup protection. Backup protection is the protection that removes the fault when the main protection or breaker refuses to act.

The action current of time-bound rapid-break over-current protection is determined by the circuit to surpass the action current of the timeless over-current protection of the adjacent line as formula (3) [1].

$$
I_{o p 1}^{\mathrm{II}}=K_{r e l} I_{o p 2}^{\mathrm{I}}
$$

$I^{\mathrm{II}}{ }_{o p 1}$ is the action current of time-bound rapid-break over-current protection (stage II protection); $K_{r e l}$ is the reliability coefficient of time-bound rapid-break over-current protection, the number is usually $1.1 \sim 1.2 ; I_{o p 2}^{\mathrm{I}}$ is the short-circuit current of the adjacent timeless rapid-break over-current protected line (stage I protection).

\section{The determination of the operation current of definite-time over-current protection}

The operation current of definite-time over-current protection is determined by the largest current to avoid the auto startup of the protection device after the short circuits in external lines are removes as formula (4) [1] .

$$
I_{o p 1}{ }^{\mathrm{II}}=\frac{K_{r e l} K_{s t}}{K_{r e}} I_{L \cdot \max }
$$

$K_{\text {rel }}$ is the reliability coefficient of the definite-time over-current protection, usually the number is $1.15 \sim 1.25 ; K_{r e}$ is the return coefficient of relays, usually the number is $0.8 \sim 0.85 ; K_{s t}$ is the auto startup coefficient of the protection device, the number is usually $1.5 \sim 3 ; I_{L \text {.max }}$ is the largest auto startup current of the protection device; $I^{\mathrm{III}}{ }_{o p 1}$ is the action current of the definite-time over-current protection (stage III protection). Usually, it can not only protection the full length of this power line, but can also protect the full length of the adjacent power line after it by working as a backup.

\section{Simulation Examples}

Virtual three-stage over-current protection experiment was performed using MATLAB simulation with the example of a $110 \mathrm{kV}$ single power supply radiant power line.

\section{A. Simulation algorithm}

The basic principles of power line relay protection are based on comparing the change of current and voltage under normal situation and fault conditions, and most protections are achieved based on fundamental components. However, due to the existence of large amount of harmonics and non-periodic components, it is hard for the protection devices to determine the action, thus in this three-stage over-current protection simulation system, we must adopt a filter algorithm to eliminate the influences of these components. In this paper, a full-wave Fourier algorithm was used in three-stage over-current protection algorithm simulation system.

Full-wave Fourier algorithm comes from the Fourier series, and it uses the orthogonal functions of sine and cosine functions to extract component signals of a certain frequency. Assume that the sampled analog signal is a periodic function of time and contains attenuated DC components as well as harmonics of different frequencies, then it can be expressed as formula (5) [5]-[9].

$$
x(t)=\sum_{\kappa=0}^{\infty}\left[a_{k} \sin \left(k \omega_{1} t\right)+b_{k} \cos \left(k \omega_{1} t\right)\right]
$$

In which, $k$, is natural number $(0,1,2, \ldots)$. When $k$ is 0 , it stands for the DC component. $a_{k}$ and $b_{k}$ are the value of each sine and cosine components.

The basic principles of the Fourier algorithm as formula (6) [5].

$$
a_{k}=\frac{2}{T} \int_{0}^{T} x(t) \sin \left(k \omega_{1} t\right) d t
$$




$$
b_{k}=\frac{2}{T} \int_{0}^{T} x(t) \cos \left(k \omega_{1} t\right) d t
$$

After Triangle transformation, merge sine and cosine terms, the equation can be written as formula (9).

$$
x_{1}(t)=\sqrt{2} X \sin \left(\omega_{1} t+\theta_{1}\right)
$$

When using a computer calculation process, trapezoidal summation method was used instead of integral method as formula (10), (11) [5]. Consider that $N \Delta t=T, \omega_{1} t=2 k \pi / N$.

$$
\begin{aligned}
& a_{1}=\frac{1}{N}\left[2 \sum_{n=1}^{N} x_{n} \sin (n 2 \pi / N)\right] \\
& b_{1}=\frac{1}{N}\left[x_{0}+\sum_{n=1}^{N-1} x_{n} \cos (n 2 \pi / N)+x_{N}\right]
\end{aligned}
$$

From the analysis above, we showed that full-wave Fourier algorithm assumed the sampled signal was periodic, and it could be used to accurately determine the fundamental frequency components. Full-wave Fourier algorithm not only can completely filter out all kinds of integer harmonics and pure DC components, but also have certain inhibitory effects on non-integer harmonics components and exponentially decaying non-periodic components. Since the full-wave Fourier algorithm is simple but performs well, it is widely used in relay protection devices [5].

\section{B. Simulation model}

Virtual three-stage over-current protection simulation model is shown in Fig. 2(a), (b). The model is composed of a three-phase power supply source, three-phase breakers, three-phase loads, relays, and three-phase fault model to simulate faults, Fourier devices, measuring devices, oscilloscopes, etc. Power line virtual three-stage over-current protection simulation model is divided into four parts: (1) Transient rapid-break over-current protection (stage I) (subsystem module in model). The main function of this subsystem is that it should immediately trip and remove faults when power line faults occur within line I. It cannot protect the full length of the line. It is determined by comparing the current from Fourier transform module and the pre-designed relay current. Since stage I over-current protection is an instantaneous action, the delay time is short, then the final signal goes through protected outlet and is outputted to the external control terminal of the breaker. (2) Time-bound over-current protection (stage II) (subsystem 1 module in model). The main function of this subsystem is that the protection system should trip and remove faults after a certain time delay when power line faults occur within line II. It not only can protect the full length of line II, but can extend its protection to part of the adjacent line after it. The time delay of stage II is usually $0.5 \mathrm{~s}$ if the sensitivity requirement is met. (3) Definite time over-current protection (stage III) (subsystem 2 module in model). It should not only protect the full length of line III, but can also protect the full length of the adjacent line after it. Its action time needs to work coordinately with the definite time over-current protection in the adjacent line after this line. (4) Protection outlet section. The main function of this part is to AND the output of line I, II and III. When the final output is 0 , it will trip the breaker and remove the fault to achieve relay protection.

\section{Simulation parameter settings}

The initial state of the circuit breaker was set as closed, the failure time was $0.4 \sim 0.8 \mathrm{~s}$ (temporary fault) or $0.4 \sim 120 \mathrm{~s}$ (permanent fault). According to the model, the action current for the transient over-current protection was set as $178 \mathrm{~A}$, the protection range is $70 \%$ of the full length, and the action time was set to $0.005 \mathrm{~s}$. The action current for the time-bound over-current protection was set as 100A, the sensitivity is 1.5 , and the action time was set to $0.5 \mathrm{~s}$. The action current for the time-bound over-current protection was set as $20 \mathrm{~A}$, the sensitivity was 1.3 , and the action time, which had to 
coordinate with the definite time over-current protection of the adjacent line after it, was set to $1.5 \mathrm{~s}$ according to a step-by-step calculation principle.

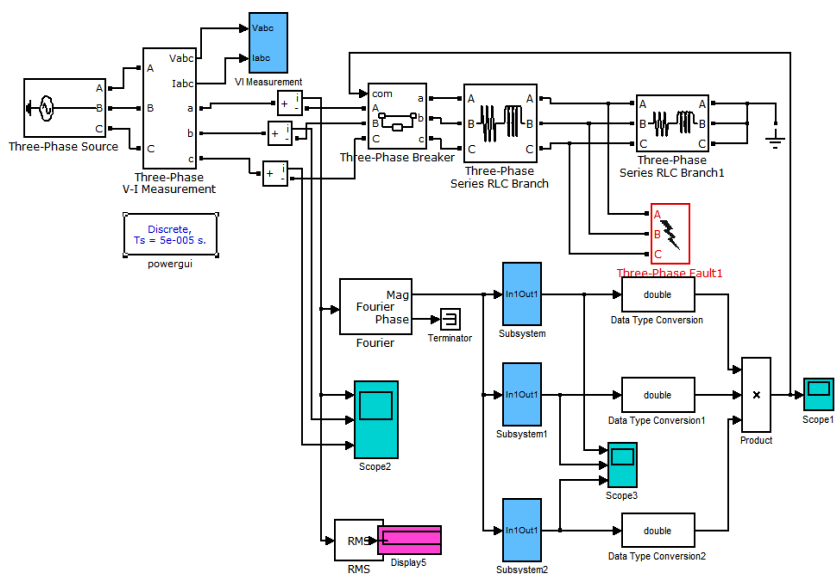

(a) simulation model

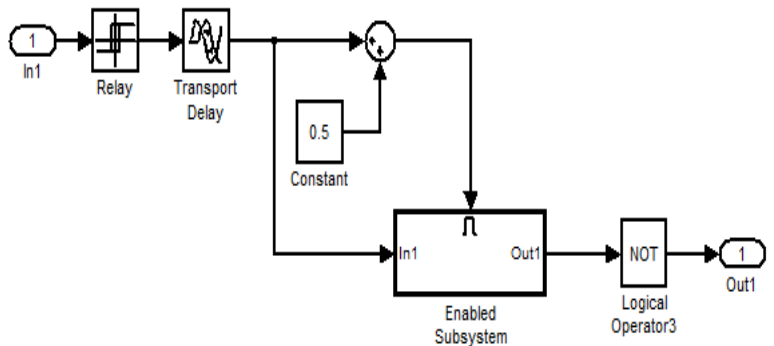

(b) three-stage subsystem

Fig. 2. Virtual three-step over-current protection model and its subsystem of three-stage

\section{Simulation Waveform Analysis}

Simulate the operating conditions and action time of each line's circuit breakers and the total circuit breaker in the three-phase over-current protected lines when there is a three-phase fault in power lines. The simulation results as shown in Fig. 3(a)-(f).

From Fig. 3(a) we can see that at $0.005 \mathrm{~s}$, stage I beaker acted and removed the fault line, thus the transient over-current protection protected the power lines. At $0.5 \mathrm{~s}$, stage II breaker acted, which means that if the breaker in stage I could not trip, stage II breaker would work as expected and removed the fault to achieve the time-bound over-current protection. Fig. 3(b) showed the waveform and action time of the total breaker.We can see that at $0.005 \mathrm{~s}$ the total breaker tripped, achieving three-stage over-current relay protection, and satisfied the requirements of rapidity, selectivity, sensitivity, and reliability for relay system.
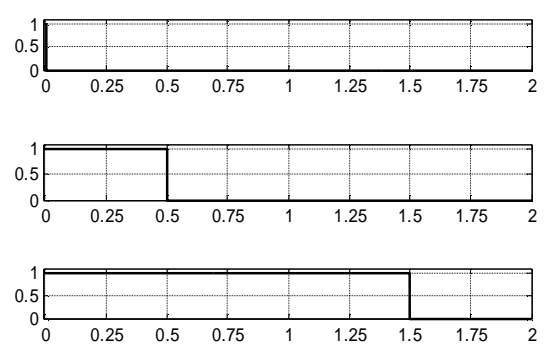

(a) breakers act waveform and time
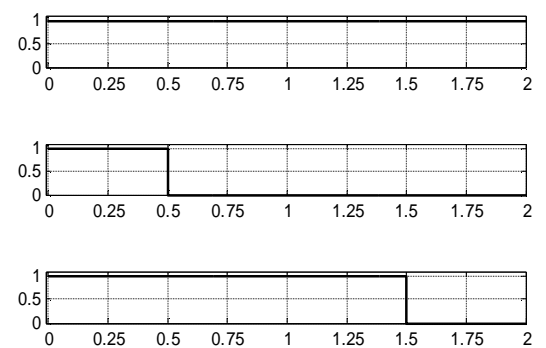

(c) breakers waveform when I stage does not act

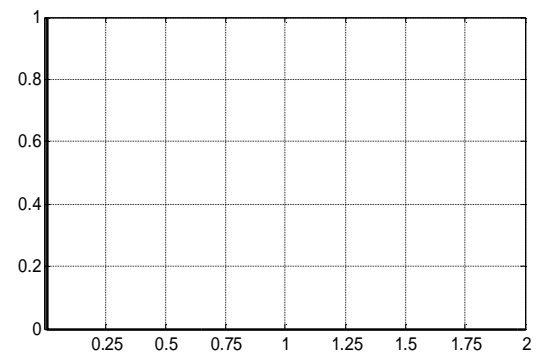

(b) total breaker acts waveform and time

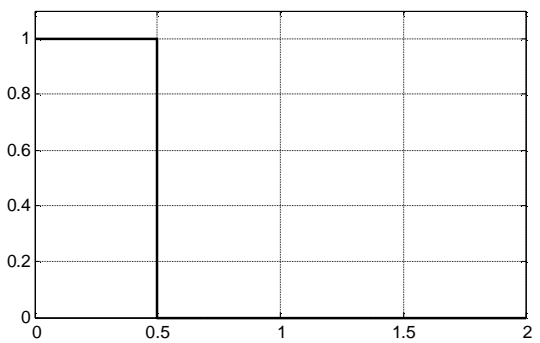

(d) total breaker act time when I stage does not act 

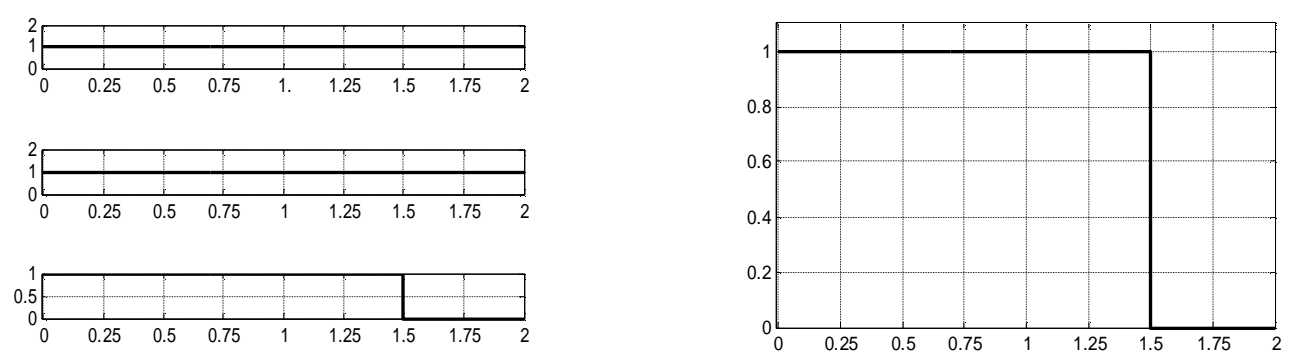

(e) breakers waveform when I, II stage do not act (f) total act time when I, II stage do not act Fig. 3 Simulation results

Figs. 3(c)-(f) simulated the waveform and action time of the breakers of each breakers and the total breaker, when there was not a tripping in stage I, or stage I and II. From Fig. 3(c)-(d), we can see that when stage I breaker did not act, the breaker in stage II and the total breaker would act at $0.5 \mathrm{~s}$ reliably, achieving their function as the main protection of this power line. From Fig. 3(e)-(f), we can see that when stage I and stage II breakers did not act, the breaker in stage III and the total breaker would act at $1.5 \mathrm{~s}$ reliably, achieving their function as the close backup of this line and the remote backup of the adjacent line after it, and satisfied the requirements of reliability for relay protection.

\section{Conclusion}

Based on the in-depth study of the fundamental theory and practical application of three-stage over-current protection, we adopted full-wave Fourier algorithm to design a three-stage over-current protection virtual simulation system, and analyzed and simulated the influences of faults, relay parameters, sensitivity, etc., of single power source supply system on the relay protection. The simulation results show that this system can be used to simulation and calculate different types of faults in the power lines, while different parameters and the dynamic process of relay protection can be observed intuitively. The analysis of results helps deepen the understanding of three-stage over-current protection, as well as provides a referable example for using MATLAB to simulate other problems of power system, and provides a reference for relevant analysis and design of the power system.

\section{References}

[1] Lihua Sun. Eletric Power Engineer Basis[M]. Beijing: China Machine Press, 2014: 178-188.

[2] Tianming wu, etc. Design and Analysis of MATLAB on Electric Power System[M]. Beijing: National Defence Industry Press, 2004.

[3] $\mathrm{Yu} \mathrm{Li}$, etc. MATLAB Application on Three-stage Over-current Protection[J]. China Electric Power Education, 2014, 33: 61-62.

[4] Jin liu. Application of MATLAB/Simpowersystem Module in Power System Analysis[J]. Sicence And Application, 2012, 11: 49-52.

[5] Qun Yu, Nan Cao. MATLAB/Simulink Model and Simulation on Electric Power System[M]. Beijing: China Machine Press, 2014.

[6] Xifan Wang, Wangliang Fang, Zhengchun Du. Electric Power System Analysis[M]. Beijing: China Science Publishing \& Media Ltd, 2003.

[7] Deshu cheng, etc. Microcomputer Relay Protection [M]. Beijing: China Electric Power Press, 2000.

[8] Chul Hwan Kim, Myung Hee Lee, Aggarwal R K. Educational use of EMTP MODELS for the study of a distance relaying algorithm for protecting transmission lines[J]. IEEE Transactions on Power Systems, 2000, 15(1): 9-15.

[9] Jie Xu, Luan Cheng. Electric Power System Relay Virtual Researcher Based on MATLAB[J]. Water Power. 2010, 36(3): 44-46. 